\title{
Parabolic Anderson model with a finite number of moving catalysts
}

\author{
F. Castell, O. Gün and G. Maillard
}

\begin{abstract}
We consider the parabolic Anderson model (PAM) which is given by the equation $\partial u / \partial t=\kappa \Delta u+\xi u$ with $u: \mathbb{Z}^{d} \times[0, \infty) \rightarrow \mathbb{R}$, where $\kappa \in[0, \infty)$ is the diffusion constant, $\Delta$ is the discrete Laplacian, and $\xi: \mathbb{Z}^{d} \times[0, \infty) \rightarrow \mathbb{R}$ is a space-time random environment. The solution of this equation describes the evolution of a "reactant" $u$ under the influence of a "catalyst" $\xi$.

In the present paper we focus on the case where $\xi$ is a system of $n$ independent simple random walks each with step rate $2 d \rho$ and starting from the origin. We study the annealed Lyapunov exponents, i.e., the exponential growth rates of the successive moments of $u$ w.r.t. $\xi$ and show that these exponents, as a function of the diffusion constant $\kappa$ and the rate constant $\rho$, behave differently depending on the dimension $d$. In particular, we give a description of the intermittent behavior of the system in terms of the annealed Lyapunov exponents, depicting how the total mass of $u$ concentrates as $t \rightarrow \infty$. Our results are both a generalization and an extension of the work of Gärtner and Heydenreich [3], where only the case $n=1$ was investigated.
\end{abstract}

F. Castell

CMI-LATP, Université de Provence, 39 rue F. Joliot-Curie, F-13453 Marseille Cedex 13, France, e-mail: castell@cmi.univ-mrs.fr

O. Gun

CMI-LATP, Université de Provence, 39 rue F. Joliot-Curie, F-13453 Marseille Cedex 13, France, e-mail: gun@cmi.univ-mrs.fr

G. Maillard

CMI-LATP, Université de Provence, 39 rue F. Joliot-Curie, F-13453 Marseille Cedex 13, France, email:maillardecmi univ-mrs. fr and EURANDOM, P.O. Box 513, 5600 MB Eindhoven, The Netherlands 


\section{Introduction}

\subsection{Model}

The parabolic Anderson model (PAM) is the partial differential equation

$$
\left\{\begin{aligned}
\frac{\partial}{\partial t} u(x, t) & =\kappa \Delta u(x, t)+\xi(x, t) u(x, t), \quad x \in \mathbb{Z}^{d}, t \geq 0 . \\
u(x, 0) & =1,
\end{aligned}\right.
$$

Here, the $u$-field is $\mathbb{R}$-valued, $\kappa \in[0, \infty)$ is the diffusion constant, $\Delta$ is the discrete Laplacian acting on $u$ as

$$
\Delta u(x, t)=\sum_{\substack{y \in \mathbb{Z}^{d} \\ y \sim x}}[u(y, t)-u(x, t)]
$$

$(y \sim x$ meaning that $y$ is nearest neighbor of $x)$, and

$$
\xi=\left(\xi_{t}\right)_{t \geq 0} \quad \text { with } \quad \xi_{t}=\left\{\xi(x, t): x \in \mathbb{Z}^{d}\right\}
$$

is an $\mathbb{R}$-valued random field that evolves with time and that drives the equation.

One interpretation of (1) comes from population dynamics by considering a system of two types of particles $A$ and $B$. $A$-particles represent "catalysts", $B$-particles represent "reactants" and the dynamics is subject to the following rules:

- A-particles evolve independently of $B$-particles according to a prescribed dynamics with $\xi(x, t)$ denoting the number of $A$-particles at site $x$ at time $t$;

- $B$-particles perform independent simple random walks at rate $2 d \kappa$ and split into two at a rate that is equal to the number of $A$-particles present at the same location;

- the initial configuration of $B$-particles is that there is exactly one particle at each lattice site.

Then, under the above rules, $u(x, t)$ represents the average number of $B$-particles at site $x$ at time $t$ conditioned on the evolution of the $A$-particles.

It is possible to add that $B$-particles die at rate $\delta \in[0, \infty)$. This leads to the trivial transformation $u(x, t) \rightarrow u(x, t) e^{\delta t}$. We will hereafter assume that $\delta=0$. It is also possible to add a coupling constant $\gamma \in(0, \infty)$ in front of the $\xi$-term in (1), but this can be reduced to $\gamma=1$ by a scaling argument.

In what follows, we focus on the case where

$$
\xi(x, t)=\sum_{k=1}^{n} \delta_{x}\left(Y_{k}^{\rho}(t)\right)
$$

with $\left\{Y_{k}^{\rho}: 1 \leq k \leq n\right\}$ a family of $n$ independent simple random walks, where for each $k \in\{1, \ldots, n\}, Y_{k}^{\rho}=\left(Y_{k}^{\rho}(t)\right)_{t \geq 0}$ is a simple random walk with step rate $2 d \rho$ 
starting from the origin. We write $\mathbb{P}_{0}^{\otimes n}$ and $\mathbb{E}_{0}^{\otimes n}$ to denote respectively the law and the expectation of the family of $n$ independent simple random walks $\left\{Y_{k}^{\rho}: 1 \leq k \leq n\right\}$ where initially all of the walkers are located at 0 .

The rest of the section is organized as follows. In Section 1.2, we define the annealed Lyapunov exponents and introduce the intermittency phenomenon. In Section 1.3. we review some related models from the literature. In Section 1.5, we state our main results, and finally, in Section 1.4 we give some further comments and add few results and conjectures.

\subsection{Lyapunov exponents and intermittency}

Our focus will be on the annealed Lyapunov exponents that describe the exponential growth rate of the successive moments of the solution of (1).

By the Feynman-Kac formula, the solution of (1) reads

$$
u(x, t)=\mathrm{E}_{x}\left(\exp \left[\int_{0}^{t} \xi\left(X^{\kappa}(s), t-s\right) d s\right]\right),
$$

where $X^{\kappa}=\left(X^{\kappa}(t)\right)_{t \geq 0}$ is the simple random walk on $\mathbb{Z}^{d}$ with step rate $2 d \kappa$ and $\mathrm{E}_{x}$ denotes expectation with respect to $X^{\kappa}$ given $X^{\kappa}(0)=x$. The connection between the parabolic Anderson equation (11) with random time-independent potential $\xi$ and the Feyman-Kac functional (3) is well understood (see e.g. Gärtner and Molchanov [10]) and can be easily extended to the time-dependent potential setting. Taking into account our choice of catalytic medium in (2) we define $\Lambda_{p}(t)$ as

$$
\begin{aligned}
\Lambda_{p}(t) & =\frac{1}{t} \log \mathbb{E}_{0}^{\otimes n}\left([u(x, t)]^{p}\right)^{1 / p} \\
& =\frac{1}{p t} \log \left(\mathbb{E}_{0}^{\otimes n} \otimes \mathbb{E}_{x}^{\otimes p}\right)\left(\exp \left[\sum_{j=1}^{p} \sum_{k=1}^{n} \int_{0}^{t} \delta_{0}\left(X_{j}^{\kappa}(s)-Y_{k}^{\rho}(t-s)\right) d s\right]\right),
\end{aligned}
$$

where $\left\{X_{j}^{\kappa}: 1 \leq j \leq p\right\}$ is a family of $p$ independent copies of $X^{\kappa}$ and $\mathrm{E}_{x}^{\otimes p}$ stands for the expectation of this family with $X_{j}^{\kappa}(0)=x$ for all $j$.

If the last quantity admits a limit as $t \rightarrow \infty$ we define

$$
\lambda_{p}:=\lim _{t \rightarrow \infty} \Lambda_{p}(t)
$$

to be the $p$-th (annealed) Lyapunov exponent of the solution $u$ of the PAM (11).

We will see in Theorem 1.1 that the limit in (5) exists and is independent of $x$. Hence, we suppress $x$ in the notation. However, $\lambda_{p}$ is clearly a function of $n, d, \kappa$ and $\rho$. In what follows, our main focus will be to analyze the dependence of $\lambda_{p}$ on the parameters $n, p, \kappa$ and $\rho$, therefore we will often write $\lambda_{p}^{(n)}(\kappa, \rho)$.

In particular, our main subject of interest will be to draw the qualitative picture of intermittency for these systems. First, note that by the moment inequality we have 


$$
\lambda_{p}^{(n)} \geq \lambda_{p-1}^{(n)}
$$

for all $p \in \mathbb{N} \backslash\{1\}$. The system (or the solution of the system) (1) is said to be p-intermittent if the above inequality is strict, namely,

$$
\lambda_{p}^{(n)}>\lambda_{p-1}^{(n)} .
$$

The system is fully intermittent if (7) holds for all $p \in \mathbb{N} \backslash\{1\}$. We will sometimes say that the system is partially intermittent if it is $p$-intermittent for some $p \in \mathbb{N} \backslash$ $\{1\}$.

Also note that, using Hölder's inequality, $p$-intermittency implies $q$-intermittency for all $q \geq p$ (see e.g. [3], Lemma 3.1). Thus, for any fixed $n \in \mathbb{N}, p$-intermittency in fact implies that

$$
\lambda_{q}^{(n)}>\lambda_{q-1}^{(n)} \quad \forall q \geq p,
$$

and 2-intermittency means full intermittency.

Geometrically, intermittency corresponds to the solution being asymptotically concentrated on a thin set, which is expected to consist of "islands" located far from each other (see [9], Section 1 and references therein for more details). Here, due to the lack of ergodicity, such a geometric picture of intermittency is not available. Nevertheless, (7) can still be interpreted as the $p$-th moment of $u$ being generated by some exponentially rare event (see [3], Section 1.2 for a more detailed analysis).

\subsection{Literature}

The behavior of the annealed Lyapunov exponents and particularly the problem of intermittency for the PAM in a space-time random environment was subject to various studies. Carmona and Molchanov [2] obtained an essentially complete qualitative description of the annealed Lyapunov exponents and intermittency when $\xi$ is white noise, i.e.,

$$
\xi(x, t)=\frac{\partial}{\partial t} W(x, t),
$$

where $W=\left(W_{t}\right)_{t \geq 0}$ with $W_{t}=\left\{W(x, t): x \in \mathbb{Z}^{d}\right\}$ is a field of independent Brownian motions. In particular, it was shown that $\lambda_{1}=1 / 2$ for all $d \geq 1$ and, $\lambda_{p}>1 / 2$ for $p \in \mathbb{N} \backslash\{1\}$ in $d=1,2$. It is also proved that for $d \geq 3$ there exist $0<\kappa_{2} \leq \kappa_{3} \leq \ldots$ satisfying

$$
\lambda_{p}(\kappa)-\frac{1}{2}\left\{\begin{array}{l}
>0, \text { for } \kappa \in\left[0, \kappa_{p}\right), \\
=0, \text { for } \kappa \in\left[\kappa_{p}, \infty\right),
\end{array} \quad p \in \mathbb{N} \backslash\{1\} .\right.
$$

Further refinements on the behavior of the Lyapunov exponents were obtained in Greven and den Hollander [11]. Upper and lower bounds on $\kappa_{p}$ were derived, and the asymptotics of $\kappa_{p}$ as $p \rightarrow \infty$ was computed. In addition, it was proved that the $\kappa_{p}$ 's are distinct for $d$ large enough. 
More recently various models where $\xi$ is non-Gaussian were investigated. Kesten and Sidoravicius [13] and Gärtner and den Hollander [4] considered the case where $\xi$ is given by a Poisson field of independent simple random walks. In [13], the survival versus extinction of the system is studied. In [4], the moment asymptotics were studied and a partial picture of intermittency, depending on the parameters $d$ and $\kappa$, was obtained. The case where $\xi$ is a single random walk -corresponding to $n=1$ case in our setting- was studied by Gärtner and Heydenreich [3]. Analogous results to those contained in Theorems 1.1, 1.2 and Corollary 1.1 (i) were obtained.

The investigation of annealed Lyapunov behavior and intermittency was extented to non-Gaussian and space correlated potentials in Gärtner, den Hollander and Maillard, in [5] and [7], for the case where $\xi$ is an exclusion process with symmetric random walk transition kernel, starting form a Bernoulli product measure. Later Gärtner, den Hollander and Maillard [8], and Maillard, Mountford and Schöpfer [14], studied the case where $\xi$ is a voter model starting either from Bernoulli product measure or from equilibrium (see Gärtner, den Hollander and Maillard [6], for an overview).

\subsection{Main results}

Our first theorem states that the Lyapunov exponents exist and behave nicely as a function of $\kappa$ and $\rho$. It will be proved in Section 2 .

Theorem 1.1 (Existence and first properties). Let $d \geq 1$ and $n, p \in \mathbb{N}$.

(i) For all $\kappa, \rho \in[0, \infty)$, the limit in (5) exists, is finite, and is independent of $x$ if $(\kappa, \rho) \neq(0,0)$.

(ii) On $[0, \infty)^{2},(\kappa, \rho) \mapsto \lambda_{p}^{(n)}(\kappa, \rho)$ is continuous, convex and non-increasing in both $\kappa$ and $\rho$.

Let $G_{d}(x)$ be the Green function at lattice site $x$ of simple random walk stepping at rate $2 d$ and

$$
\mu(\kappa)=\sup \operatorname{Sp}\left(\kappa \Delta+\delta_{0}\right)
$$

be the supremum of the spectrum of the operator $\kappa \Delta+\delta_{0}$ in $l^{2}\left(\mathbb{Z}^{d}\right)$. It is well-known that (see e.g. [4], Lemma 1.3) $\operatorname{Sp}\left(\kappa \Delta+\delta_{0}\right)=[-4 d \kappa, 0] \cup\{\mu(\kappa)\}$ with

$$
\mu(\kappa) \begin{cases}=0, & \text { if } \kappa \geq G_{d}(0), \\ >0, & \text { if } \kappa<G_{d}(0) .\end{cases}
$$

Furthermore, $\kappa \mapsto \mu(\kappa)$ is continuous, non-increasing and convex on $[0, \infty)$, and strictly decreasing on $\left[0, G_{d}(0)\right]$.

The next theorem gives the limiting behavior of $\lambda_{p}^{(n)}$ as $\kappa \downarrow 0$ and $\kappa \rightarrow \infty$, and describes a region of $\kappa$ where $\lambda_{p}^{(n)}=0$. Note that by symmetry, $\lambda_{p}^{(n)}(\kappa, \rho)=$ $\frac{n}{p} \lambda_{n}^{(p)}(\rho, \kappa)$, for all $n, p \in \mathbb{N}$ and $\kappa, \rho \in[0, \infty)$. Therefore, the $\kappa$-dependence described below can be transcribed in terms of $\rho$-dependence. 
Theorem 1.2 ( $\kappa$ - $\rho$-dependence). Let $n, p \in \mathbb{N}$ and $\rho \in[0, \infty)$.

(i) For all $d \geq 1, \lim _{\kappa \downarrow 0} \lambda_{p}^{(n)}(\kappa, \rho)=\lambda_{p}^{(n)}(0, \rho)=n \mu(\rho / p)$.

(ii) If $1 \leq d \leq 2$, then $\lambda_{p}^{(n)}(\kappa, \rho)>0$ for all $\kappa \in[0, \infty)$. Moreover, $\kappa \mapsto \lambda_{p}^{(n)}(\kappa, \rho)$ is strictly decreasing with $\lim _{\kappa \rightarrow \infty} \lambda_{p}^{(n)}(\kappa, \rho)=0$ (see Fig. 1).

(iii) If $d \geq 3$, then $\lambda_{p}^{(n)}(\kappa, \rho)=0$ for all $\kappa \in\left[n G_{d}(0), \infty\right)$ (see Fig. 22).

Our next result describes the limiting behavior of $\lambda_{p}^{(n)}$ as $p \rightarrow \infty$ and $n \rightarrow \infty$.

Theorem 1.3 ( $n$ - $p$-dependence). Let $d \geq 1$ and $\kappa, \rho \in[0, \infty)$.

(i) For all $n \in \mathbb{N}, \lim _{p \rightarrow \infty} \lambda_{p}^{(n)}(\kappa, \rho)=n \mu(\kappa / n)$ (see Fig. 11 2);

(ii) For all $p>\rho / G_{d}(0), \lim _{n \rightarrow \infty} \lambda_{p}^{(n)}(\kappa, \rho)=+\infty$;

(iii) For all $p \leq \rho / G_{d}(0)$ and $n \in \mathbb{N}, \lambda_{p}^{(n)}(\kappa, \rho)=0$.

By part (ii) of Theorem $1.1 \lambda_{p}^{(n)}(\kappa, \rho)$ is non-increasing in $\kappa$. Hence, we can define $\left\{\kappa_{p}^{(n)}(\rho): p \in \mathbb{N}\right\}$ as the non-decreasing sequence of critical $\kappa$ 's for which

$$
\lambda_{p}^{(n)}(\kappa, \rho)\left\{\begin{array}{l}
>0, \text { for } \kappa \in\left[0, \kappa_{p}^{(n)}(\rho)\right), \\
=0, \text { for } \kappa \in\left[\kappa_{p}^{(n)}(\rho), \infty\right),
\end{array} \quad p \in \mathbb{N} .\right.
$$

As a consequence of Theorems 1.1 and 1.2 we have,

$$
\begin{cases}\kappa_{p}^{(n)}(\rho)=\infty, & \text { if } 1 \leq d \leq 2, \\ 0<\kappa_{p}^{(n)}(\rho)<\infty, & \text { if } d \geq 3 \text { and } p>\rho / G_{d}(0), \\ \kappa_{p}^{(n)}(\rho)=0, & \text { if } d \geq 3 \text { and } p \leq \rho / G_{d}(0) .\end{cases}
$$

Our fourth theorem, which gives bounds on $\kappa_{p}^{(n)}(\rho)$ for $d \geq 3$, will be proved in Section 4 For this theorem we need to define the inverse of the function $\mu(\kappa)$. Note that by (8) and (9) we have $\mu(0)=1$ and $\mu\left(G_{d}(0)\right)=0$. It is easy to see that $\mu(\kappa)$ restricted to the domain $\left[0, G_{d}(0)\right]$ is invertible with an inverse function $\mu^{-1}:[0,1] \rightarrow\left[0, G_{d}(0)\right]$. We extend $\mu^{-1}$ to $[0, \infty)$ by declaring $\mu^{-1}(t)=0$ for $t>1$. Denote

$$
\alpha_{d}=\frac{G_{d}(0)}{2 d\left\|G_{d}\right\|_{2}^{2}} \in[0, \infty),
$$

where $\left\|G_{d}\right\|_{2}$ is the $l_{2}$ norm of $G_{d}$. Since $\left\|G_{d}\right\|_{2}<\infty$ if and only if $d \geq 5, \alpha_{d}=0$ for $d \in\{3,4\}$.

Theorem 1.4 (Critical $\kappa$ 's). Let $n, p \in \mathbb{N}$.

(i) If $d \geq 3$, then $\rho \in[0, \infty) \mapsto \kappa_{p}^{(n)}(\rho)$ is a continuous, non-increasing and convex function such that

$$
\max \left(\frac{n}{4 d} \mu(\rho / p), n \mu^{-1}(4 d \rho / p)\right) \leq \kappa_{p}^{(n)}(\rho) \leq n G_{d}(0)\left(1-\frac{\rho}{p G_{d}(0)}\right)_{+} .
$$

(ii) If $d \geq 5$, then 


$$
\kappa_{p}^{(n)}(\rho) \geq\left(n G_{d}(0)-\rho \frac{n}{p \alpha_{d}}\right)_{+} .
$$

(iii) If $d \geq 5$ and $p \in \mathbb{N} \backslash\{1\}$ are such that $\alpha_{d}>\frac{p-1}{p}$, then

$$
\kappa_{p-1}^{(n)}(\rho)<\kappa_{p}^{(n)}(\rho) \quad \forall \rho \in\left(0, p G_{d}(0)\right)
$$

Note that the condition $\alpha_{d}>\frac{p-1}{p}$ is always true if $d$ is large enough by the following lemma, whose proof is given in the appendix.

Lemma 1.1. If $d \geq 3$, then $\alpha_{d} \leq 1$ and $\lim _{d \rightarrow \infty} \alpha_{d}=1$.

As a consequence of the previous statements, our next result gives some general intermittency properties for all dimensions, and describes several regimes in the intermittent behavior of the system.

Corollary 1.1 (Intermittency). Let $n \in \mathbb{N}$.

(i) If $d \geq 1$, then (see Fig. 2)

- for $\kappa \in\left[0, n G_{d}(0)\right)$ there exists $p \geq 2$ such that the system is p-intermittent;

- for $\kappa \in\left[n G_{d}(0), \infty\right)$ the system is not intermittent.

(ii) Fix $p \in \mathbb{N} \backslash\{1\}$. If $d$ is large enough (such that $\alpha_{d}>(p-1) / p$ ), then for all $q \in\{2, \ldots, p\}, \rho \in\left[0, q G_{d}(0)\right)$ and $\kappa \in\left(\kappa_{q-1}^{(n)}(\rho), \kappa_{q}^{(n)}(\rho)\right)$, the system is $q$ intermittent (see Fig. 3).

Note that since $G_{d}(0)=\infty$ for $d=1,2$ Corollary 1.1 (i) implies that for dimensions 1 and 2 the system is always $p$-intermittent for some $p$. Some other partial results about intermittency are given in section 1.5 (see also figures).

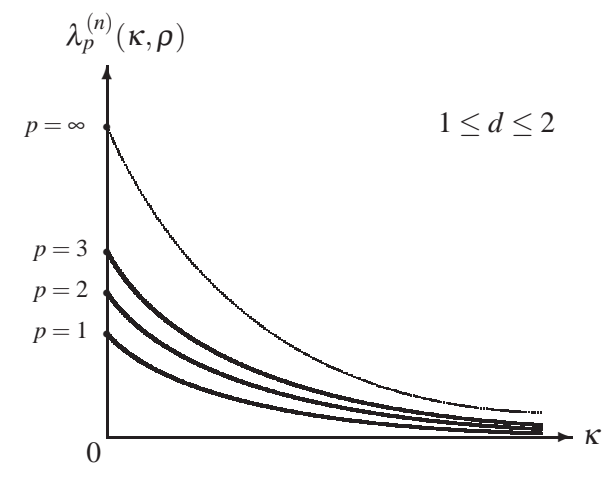

Fig. 1 For $1 \leq d \leq 2$, the system is partially intermittent. Full intermittency is conjectured, and proved for $n=1,2$. 


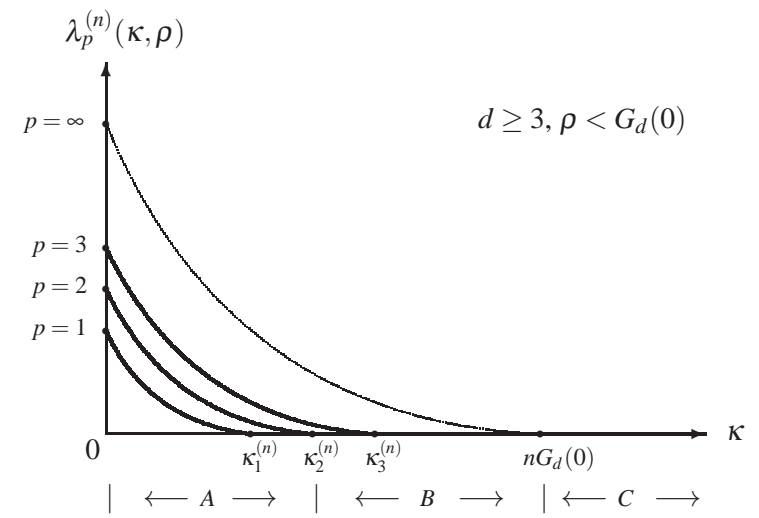

Fig. 2 For $d \geq 3$ and $\rho<G_{d}(0)$, the system is partially intermittent on $A \cup B$ and not intermittent on $C$. Full intermittency on $A$ is conjectured, and proved for $n=1,2$.

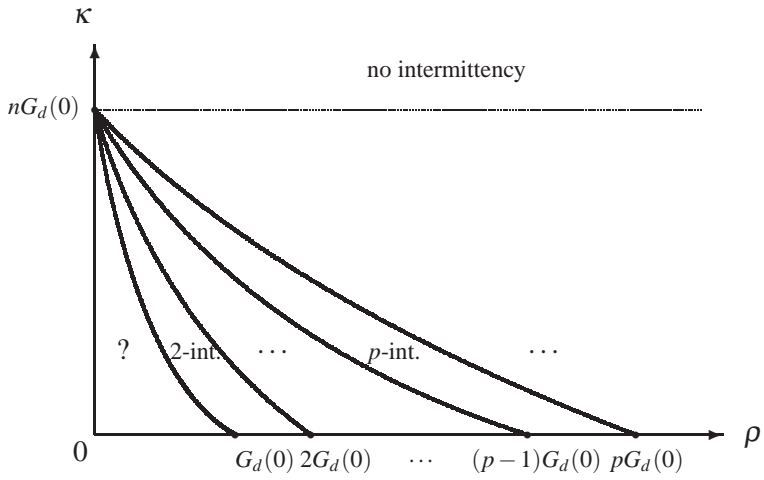

Fig. 3 Phase diagram of intermittency when $d$ is large enough. The bold curves represent $\rho \in$ $[0, \infty) \mapsto \kappa_{q}^{(n)}(\rho), q=1, \cdots, p$. In the "?" region, full intermittency is proved in a small neighborhood of 0 .

\subsection{Discussion}

Our results can be extended to various different random medium. For example, consider the system of catalysts given by a collection of independent random walks where there is one walker starting from each site of a large box. More precisely, let $D_{R}$ denote the box in $\mathbb{Z}^{d}$ with side length $R$. Consider the random medium

$$
\xi(x, t)=\sum_{k \in D_{R}} \delta_{x}\left(Y_{k}^{\rho}(t)\right)
$$


with $\left\{Y_{k}^{\rho}: k \in D_{R}\right\}$ a family of $R^{d}$ simple random walks, where for each $k \in D_{R}$, $Y_{k}^{\rho}$ is a simple random walk with step rate $2 d \rho$ starting from $Y_{k}^{\rho}(0)=k$. For a fixed size box, there is a positive probability that all the random walks meet at the origin in finite time. Then, it is easy to see that the Lyapunov exponents are the same as in the case of $n$ independent random walks starting from the origin where $n=R^{d}$. An interesting set up would be case where the length of the initial box grows with time. A natural question arises as whether the large time limit would be related to the case of Poisson field of simple random walks, considered in [4], or it would have different behavior depending on how fast the size of the box grows with time.

Let us now discuss some facts about the intermittent picture. First of all, as one can easily guess from (4), $\lambda_{p}^{(n)}(\kappa, \rho)$ is the top of the spectrum of the operator $\mathscr{L}_{p}$ where for $f\left(x_{1}, \cdots, x_{p}, y_{1}, \cdots, y_{n}\right)$ in $l_{2}\left(\mathbb{Z}^{d(p+n)}\right) \mathscr{L}_{p}$, is defined by:

$$
\mathscr{L}_{p}(f)=\kappa \sum_{k=1}^{p} \Delta_{x_{k}} f+\rho \sum_{j=1}^{n} \Delta_{y_{j}} f+I_{p} f .
$$

Here

$$
I_{p} f\left(x_{1}, \cdots, x_{p}, y_{1}, \cdots, y_{n}\right)=\sum_{k=1}^{n} \sum_{j=1}^{p} \delta_{0}\left(x_{j}-y_{k}\right) f\left(x_{1}, \cdots, x_{p}, y_{1}, \cdots, y_{n}\right) .
$$

This is the meaning of equation (20) of Section 2 from which most of our results are derived. The following proposition links full intermittency and existence of an eigenfunction corresponding to $\lambda_{1}^{(n)}(\kappa, \rho)$.

Proposition 1.1. If there exists $f \in l_{2}\left(\mathbb{Z}^{d(1+n)}\right)$ with $\|f\|_{2}=1$, such that $\mathscr{L}_{1}(f)=$ $\lambda_{1}^{(n)}(\kappa, \rho) f$, then $\lambda_{2}^{(n)}(\kappa, \rho)>\lambda_{1}^{(n)}(\kappa, \rho)$, and the system is fully intermittent.

Proposition 1.1 is proved in the appendix. The existence of an eigenfunction corresponding to $\lambda_{1}^{(n)}(\kappa, \rho)$ (and therefore full intermittency) was proved in the following cases:

- $n=1,2$ and $\kappa+\rho<n G_{d}(0)$. This is done in [3] for $n=1$, and in [15] for $n=2$.

- $n \geq 3$ and $4 d(\rho n+\kappa)<1$ in [15].

To prove these results, in [3] and [15] $\lambda_{1}^{(n)}(\kappa, \rho)$ was expressed as the top of the spectrum of the operator $\mathscr{H}=\mathscr{B}+\sum_{j=1}^{n} \delta_{0}\left(z_{j}\right)$, where $\mathscr{B}$ is the generator of the Markov process $Z(t)=\left(X_{1}^{\kappa}(t)-Y_{1}^{\rho}(t), \cdots, X_{1}^{\kappa}(t)-Y_{n}^{\rho}(t)\right)$ (see (4)). For $n=1, \mathscr{H}$ is just $(\kappa+\rho) \Delta+\delta_{0}$, which is a compact perturbation of $(\kappa+\rho) \Delta$. This fact easily implies the existence of an eigenfunction corresponding to $\lambda_{1}^{(1)}(\kappa, \rho)$. However, this is no more the case as soon as $n \geq 2$. In [15], Schnitzler and Wolff considered $\mathscr{B}$ as a perturbation of $\sum_{j=1}^{n} \delta_{0}\left(z_{j}\right)$, leading to the results for $n \geq 2$. Expressing $\lambda_{p}^{(n)}(\kappa, \rho)$ in terms of the process $(Z(t))_{t \geq 0}$ does not seem very fruitful in cases other than the one treated in [3] and[15]. Therefore, it appeared to us more natural and more tractable to express $\lambda_{p}^{(n)}(\kappa, \rho)$ in terms of the process $\left(X_{1}^{\kappa}(t), \cdots, X_{p}^{\kappa}(t), Y_{1}^{\rho}(t), \cdots, Y_{n}^{\rho}(t)\right)$. We complete the intermittent picture by the following conjecture: 
Conjecture 1.1 (Intermittency). Fix $n \in \mathbb{N}$. Then (see Fig. 1, 2),

(i) for $1 \leq d \leq 2$, the system is full intermittent (proved for $n=1,2$ );

(ii) for $d \geq 3$, the intermittency vanishes as $\kappa$ increases. More precisely, for $d \geq 3$, there are three different regimes:

A: for $\kappa \in\left[0, \kappa_{2}^{(n)}\right.$ ), the system is full intermittent (proved in a small neighborhood of 0 );

B: for $\kappa \in\left[\kappa_{2}^{(n)}, n G_{d}(0)\right)$, there exists $p=p(\kappa) \geq 3$ such that the system is $q$ intermittent for all $q \geq p$;

C: for $\kappa \in\left[n G_{d}(0), \infty\right)$, the system is not $p$-intermittent for any $p \geq 2$.

To complete Theorem 1.4, we close with a conjecture about critical $\kappa$ 's, whose analogue for white noise potential was conjectured in Carmona and Molchanov [2] and partially proved in Greven and den Hollander [11]:

Conjecture 1.2 (Critical $\kappa$ 's). For all fixed $n \geq 1$ and $d$ large enough the $\kappa_{p}^{(n)}$,s are distinct (see Fig. 22).

\section{Proof of Theorem 1.1}

Step 1: We first prove that if the limit in (5) exists for $x=0$, then it exists for all $x \in \mathbb{Z}^{d}$ and does not depend on $x$ as soon as $(\kappa, \rho) \neq(0,0)$. To this end, let us introduce some notations. For any $t>0$, we denote

$$
Y_{t}=\left(Y_{1}^{\rho}(t), \cdots, Y_{n}^{\rho}(t)\right) \in \mathbb{Z}^{d n}, X_{t}=\left(X_{1}^{\kappa}(t), \cdots, X_{p}^{\kappa}(t)\right) \in \mathbb{Z}^{d p} .
$$

For $(x, y) \in \mathbb{Z}^{d p} \times \mathbb{Z}^{d n}, \mathbb{E}_{x, y}^{X, Y}$ denote the expectation under the law of $\left(X_{t}, Y_{t}\right)_{t \geq 0}$ starting from $(x, y)$. The same notation is used for $x \in \mathbb{Z}^{d}$ and $y \in \mathbb{Z}^{d}$. In that case, it means that $X_{0}=(x, \cdots, x), Y_{0}=(y, \cdots, y)$ and $\mathbb{E}_{x, y}^{X, Y}=\mathbb{E}_{y}^{\otimes n} \otimes \mathrm{E}_{x}^{\otimes p}$. Finally, for $x=\left(x_{1}, \cdots, x_{p}\right) \in \mathbb{Z}^{d p}$ and $y=\left(y_{1}, \cdots, y_{n}\right) \in \mathbb{Z}^{d n}$, set

$$
I_{p}(x, y)=\sum_{j=1}^{p} \sum_{k=1}^{n} \delta_{0}\left(x_{j}-y_{k}\right)
$$

Then, by time reversal for $Y$ in (4), for all $x \in \mathbb{Z}^{d}$ and $t>0$,

$$
\mathbb{E}_{0}^{\otimes n}\left[u(x, t)^{p}\right]=\sum_{z \in \mathbb{Z}^{d n}} \mathbb{E}_{x, z}^{X, Y}\left[\exp \left(\int_{0}^{t} I_{p}\left(X_{s}, Y_{s}\right) d s\right) \delta_{0}\left(Y_{t}\right)\right] .
$$

Using the Markov property at time 1 and the fact that $1 \leq \exp \left(\int_{0}^{1} I_{p}\left(X_{s}, Y_{s}\right) d s\right)$, we get for $x_{1}$ and $x_{2}$ any fixed points in $\mathbb{Z}^{d}$, 


$$
\begin{aligned}
\mathbb{E}_{0}^{\otimes n}\left[u\left(x_{1}, t\right)^{p}\right] & \geq \sum_{z \in \mathbb{Z}^{d n}} \mathbb{E}_{x_{1}, z}^{X, Y}\left[\delta_{\left(x_{2}, \cdots, x_{2}\right)}\left(X_{1}\right) \delta_{z}\left(Y_{1}\right) \exp \left(\int_{1}^{t} I_{p}\left(X_{s}, Y_{s}\right) d s\right) \delta_{0}\left(Y_{t}\right)\right] \\
& =\left(p_{1}^{\kappa}\left(x_{1}, x_{2}\right)\right)^{p}\left(p_{1}^{\rho}(0,0)\right)^{n} \mathbb{E}_{0}^{\otimes n}\left(\left[u\left(x_{2}, t-1\right)\right]^{p}\right),
\end{aligned}
$$

where $p_{t}^{v}$ is the transition kernel of a simple random walk on $\mathbb{Z}^{d}$ with step rate $2 d v$. This proves the independence of $\lambda_{p}$ w.r.t. $x$ as soon as $\kappa>0$, since in this case for all $x_{1}, x_{2} \in \mathbb{Z}^{d}, p_{1}^{\kappa}\left(x_{1}, x_{2}\right)>0$.

For $\kappa=0$, since the $X$-particles do not move, we have

$$
\mathbb{E}_{0}^{\otimes n}\left[u\left(x_{1}, t\right)^{p}\right]=\mathbb{E}_{0}\left[\exp \left(p \int_{0}^{t} \delta_{x_{1}}\left(Y_{1}^{\rho}(s)\right) d s\right)\right]^{n} .
$$

The same reasoning leads now to

$$
\mathbb{E}_{0}^{\otimes n}\left[u\left(x_{1}, t\right)^{p}\right] \geq p_{1}^{\rho}\left(0, x_{1}-x_{2}\right)^{n} \mathbb{E}_{0}^{\otimes n}\left(\left[u\left(x_{2}, t-1\right)\right]^{p}\right) .
$$

Step 2: Variational representation. From now on, we restrict our attention to the case $x=0$. The aim of this step is to give a variational representation of $\lambda_{p}^{(n)}(\kappa, \rho)$. To this end, we introduce further notations. Let $\left(e_{1}, \cdots, e_{d}\right)$ be the canonical basis of $\mathbb{R}^{d}$. For $x=\left(x_{1}, \cdots, x_{p}\right) \in \mathbb{Z}^{d p}$, and $f:(x, y) \in \mathbb{Z}^{d p} \times \mathbb{Z}^{d n} \mapsto \mathbb{R}$, we set

$$
\nabla_{x} f(x, y)=\left(\nabla_{x_{1}} f(x, y), \cdots, \nabla_{x_{p}} f(x, y)\right) \in \mathbb{R}^{d p},
$$

where for $j \in\{1, \cdots, p\}$, and $i \in\{1, \cdots, d\}$,

$$
\left\langle\nabla_{x_{j}} f(x, y), e_{i}\right\rangle=f\left(x_{1}, \cdots, x_{j}+e_{i}, \cdots, x_{p}, y\right)-f(x, y) .
$$

The same notation is used for the $y$-coordinates, so that $\nabla_{y} f(x, y) \in \mathbb{R}^{d n}$. We also define

$$
\begin{aligned}
\Delta_{x} f(x, y) & =\sum_{j=1}^{p} \Delta_{x_{j}} f(x, y) \\
& =\sum_{j=1}^{p} \sum_{\substack{z_{j} \in \mathbb{Z}^{d} \\
z_{j} \sim x_{j}}}\left[f\left(x_{1}, \cdots, z_{j}, \cdots, x_{p}, y\right)-f\left(x_{1}, \cdots, x_{j}, \cdots, x_{p}, y\right)\right] .
\end{aligned}
$$

Proposition 2.1. Let $d \geq 1$ and $n, p \in \mathbb{N}$. For all $\kappa, \rho \in[0, \infty)$,

$$
\begin{aligned}
\lambda_{p}^{(n)}(\kappa, \rho) & =\lim _{t \rightarrow \infty} \frac{1}{p t} \log \mathbb{E}_{0}^{\otimes n}\left[u(0, t)^{p}\right] \\
& =\frac{1}{p} \sup _{\substack{f \in l^{2}\left(\mathbb{Z}^{d p} \times \|^{d n}\right) \\
\|f\|_{2}=1}}\left\{-\kappa\left\|\nabla_{x} f\right\|_{2}^{2}-\rho\left\|\nabla_{y} f\right\|_{2}^{2}+\sum_{(x, y)} I_{p}(x, y) f^{2}(x, y)\right\} .
\end{aligned}
$$


Proof. Upper bound. For a positive integer $m$, let $B_{R}^{m}$ denote the ball in $\mathbb{Z}^{d m}$ of radius $R=t \log (t)$ centered at the origin. We first prove the following lemma which states we can restrict (18) to $X$ paths being in $B_{R}^{p}$ at time $t$ and $Y$ paths starting from $B_{R}^{n}$.

Lemma 2.1. As $t \rightarrow \infty$,

$$
\mathbb{E}_{0}^{\otimes n}\left[u(x, t)^{p}\right]=(1+o(1)) \sum_{z \in B_{R}^{n}} \mathbb{E}_{0, z}^{X, Y}\left[\exp \left(\int_{0}^{t} I_{p}\left(X_{s}, Y_{s}\right) d s\right) \delta_{0}\left(Y_{t}\right) \mathbb{I}_{\left(B_{R}^{p}\right)}\left(X_{t}\right)\right] .
$$

Proof. It is enough to prove that

$$
r(t):=\frac{\mathbb{E}_{0}^{\otimes n}\left[u(x, t)^{p}\right]-\sum_{z \in B_{R}^{n}} \mathbb{E}_{0, z}^{X, Y}\left[\exp \left(\int_{0}^{t} I_{p}\left(X_{s}, Y_{s}\right) d s\right) \delta_{0}\left(Y_{t}\right) \mathbb{I}_{\left(B_{R}^{p}\right)}\left(X_{t}\right)\right]}{\mathbb{E}_{0}^{\otimes n}\left[u(x, t)^{p}\right]}
$$

converges to 0 as $t \rightarrow \infty$. Using the trivial bounds

$$
1 \leq \exp \left(\int_{0}^{t} I_{p}\left(X_{s}, Y_{S}\right) d s\right) \leq \exp (\operatorname{tnp})
$$

and splitting the sum in 18), we get

$$
\begin{aligned}
r(t) & \leq \frac{e^{t n p}}{\sum_{z \in Z^{d n}} \mathbb{E}_{0, z}^{X, Y}\left[\delta_{0}\left(Y_{t}\right)\right]}\left(\sum_{z \notin B_{R}^{n}} \mathbb{E}_{0, z}^{X, Y}\left[\delta_{0}\left(Y_{t}\right)\right]+\sum_{z \in B_{R}^{n}} \mathbb{E}_{0, z}^{X, Y}\left[\delta_{0}\left(Y_{t}\right) \mathbb{I}_{\left(B_{R}^{p}\right)^{c}}\left(X_{t}\right)\right]\right) \\
& \leq \frac{e^{t n p}}{\sum_{z \in \mathbb{Z}^{d n}} \mathbb{P}_{z}\left(Y_{t}=0\right)}\left(\sum_{z \notin B_{R}^{n}} \mathbb{P}_{z}\left(Y_{t}=0\right)+\mathbb{P}_{0}\left(X_{t} \notin B_{R}^{p}\right) \sum_{z \in B_{R}^{n}} \mathbb{P}_{z}\left(Y_{t}=0\right)\right) \\
& \leq e^{t n p}\left(\mathbb{P}_{0}\left(Y_{t} \notin B_{R}^{n}\right)+\mathbb{P}_{0}\left(X_{t} \notin B_{R}^{p}\right)\right),
\end{aligned}
$$

where for the last two inequalities we used the time-reversal of $Y$. We have for $R=t \log (t)$ and large enough $t$

$$
\begin{aligned}
& \mathbb{P}_{0}\left(Y_{1}^{\rho}(t) \notin B_{R}^{1}\right) \leq \exp [-C(d, \rho) t \log (t)], \\
& \mathbb{P}_{0}\left(X_{1}^{\kappa}(t) \notin B_{R}^{1}\right) \leq \exp [-C(d, \kappa) t \log (t)]
\end{aligned}
$$

for some positive constants $C(d, \rho)$ and $C(d, \kappa)$ (see for instance Lemma 4.3 in [10]). Using this we get

$$
r(t) \leq e^{t n p}\left(n e^{-C(d, \rho) t \log t}+p e^{-C(d, \kappa) t \log t}\right) \stackrel{t \rightarrow \infty}{\longrightarrow} 0 .
$$

This finishes the proof of the lemma.

Using Lemma2.1 it is enough to study the existence of 


$$
\begin{aligned}
\lim _{t \rightarrow \infty} \frac{1}{t} \log \sum_{z \in B_{R}^{n}} \mathbb{E}_{0, z}^{X, Y}\left[\exp \left(\int_{0}^{t} I_{p}\left(X_{s}, Y_{S}\right) d s\right) \delta_{0}\left(Y_{t}\right) \mathbb{I}_{B_{R}^{p}}\left(X_{t}\right)\right] \\
\quad=\lim _{t \rightarrow \infty} \frac{1}{t} \log \left\langle f_{1}, e^{t \mathscr{L}_{p}} f_{2}\right\rangle,
\end{aligned}
$$

where $f_{1}:(x, y) \in \mathbb{Z}^{d p} \times \mathbb{Z}^{d n} \mapsto \delta_{0}(x) \mathbb{I}_{B_{R}^{n}}(y), f_{2}:(x, y) \in \mathbb{Z}^{d p} \times \mathbb{Z}^{d n} \mapsto \mathbb{I}_{B_{R}^{p}}(x) \delta_{0}(y)$, and $\mathscr{L}_{p}$ is the bounded self-adjoint operator in $l^{2}\left(\mathbb{Z}^{d p} \times \mathbb{Z}^{d n}\right)$ defined by

$$
\mathscr{L}_{p} f(x, y)=\kappa \Delta_{x} f(x, y)+\rho \Delta_{y} f(x, y)+I_{p}(x, y) f(x, y), \quad(x, y) \in \mathbb{Z}^{d p} \times \mathbb{Z}^{d n} .
$$

For a linear operator $\mathscr{L}$ on $l^{2}\left(\mathbb{Z}^{d p} \times \mathbb{Z}^{d n}\right)$ we define

$$
\|\mathscr{L}\|_{2,2}:=\sup _{\substack{f \in l^{2}\left(\mathbb{Z}^{d p} \times \mathbb{X} \mathbb{Z}^{d n}\right) \\\|f\|_{2}=1}}\langle f, \mathscr{L} f\rangle
$$

Note that we have

$$
\left\langle f_{1}, e^{t \mathscr{L}_{p}} f_{2}\right\rangle \leq\left\|f_{1}\right\|_{2}\left\|e^{t \mathscr{L}_{p}}\right\|_{2,2}\left\|f_{2}\right\|_{2}=C(d, n, p) R^{d(n+p) / 2}\left\|e^{t \mathscr{L}_{p}}\right\|_{2,2},
$$

for some constant $C(d, n, p)>0$. Thus,

$$
\lim _{t \rightarrow \infty} \frac{1}{t} \log \left\langle f_{1}, e^{t \mathscr{L}_{p}} f_{2}\right\rangle \leq\left\|\mathscr{L}_{p}\right\|_{2,2}=\sup _{\substack{f \in l^{2}\left(\mathbb{Z}^{d} p_{\times} \mathbb{Z}^{d n}\right) \\\|f\|_{2}=1}}\left\langle f, \mathscr{L}_{p} f\right\rangle,
$$

which is the upper bound in (20).

Lower bound. By (18) with $x=0$, it follows that

$$
\begin{aligned}
\mathbb{E}_{0}^{\otimes n}\left[u(0, t)^{p}\right] & \geq \mathbb{E}_{0,0}^{X, Y}\left[\exp \left(\int_{0}^{t} I_{p}\left(X_{s}, Y_{s}\right) d s\right) \delta_{0}\left(X_{t}\right) \delta_{0}\left(Y_{t}\right)\right] \\
& =\left\langle\delta_{0} \otimes \delta_{0}, e^{t \mathscr{L}_{p}}\left(\delta_{0} \otimes \delta_{0}\right)\right\rangle=\left\|e^{\frac{t}{2} \mathscr{L}_{p}}\left(\delta_{0} \otimes \delta_{0}\right)\right\|_{2}^{2} \\
& =\sum_{x \in \mathbb{Z}^{d p}} \sum_{y \in \mathbb{Z}^{d n}}\left(e^{\frac{t}{2} \mathscr{L}_{p}}\left(\delta_{0} \otimes \delta_{0}\right)(x, y)\right)^{2} .
\end{aligned}
$$

Restricting the sum over $B_{R}^{p} \times B_{R}^{n}$, and applying Jensen's inequality, we get 


$$
\begin{aligned}
\mathbb{E}_{0}^{\otimes n}\left[u(0, t)^{p}\right] & \geq \sum_{x \in B_{R}^{p}} \sum_{y \in B_{R}^{n}}\left(e^{\frac{t}{2} \mathscr{L}_{p}}\left(\delta_{0} \otimes \delta_{0}\right)(x, y)\right)^{2} \\
& \geq \frac{1}{\left|B_{R}^{n}\right|} \frac{1}{\left|B_{R}^{p}\right|}\left(\sum_{x \in B_{R}^{p}} \sum_{y \in B_{R}^{n}} e^{\frac{t}{2} \mathscr{L}_{p}}\left(\delta_{0} \otimes \delta_{0}\right)(x, y)\right)^{2} \\
& =\frac{C(d, n, p)}{R^{d(n+p)}}\left(\sum_{x \in B_{R}^{p}} \sum_{y \in B_{R}^{n}} \mathbb{E}_{x, y}^{X, Y}\left[\exp \left(\int_{0}^{t / 2} I_{p}\left(X_{s}, Y_{S}\right) d s\right) \delta_{0}\left(X_{t / 2}\right) \delta_{0}\left(Y_{t / 2}\right)\right]\right)^{2} \\
& =\frac{C(d, n, p)}{R^{d(n+p)}}\left(\mathbb{E}_{0,0}^{X, Y}\left[\exp \left(\int_{0}^{t / 2} I_{p}\left(X_{s}, Y_{s}\right) d s\right) \mathbb{I}_{B_{R}^{p}}\left(X_{t / 2}\right) \mathbb{I}_{B_{R}^{n}}\left(Y_{t / 2}\right)\right]\right)^{2} .
\end{aligned}
$$

Taking $R=t \log (t)$, we obtain that

$$
\begin{aligned}
\liminf _{t \rightarrow \infty} & \frac{1}{t} \log \mathbb{E}_{0}^{\otimes n}\left[u(0, t)^{p}\right] \\
& \geq \liminf _{t \rightarrow \infty} \frac{2}{t} \log \mathbb{E}_{0,0}^{X, Y}\left[\exp \left(\int_{0}^{t / 2} I_{p}\left(X_{s}, Y_{S}\right) d s\right) \mathbb{I}_{B_{R}^{p}}\left(X_{t / 2}\right) \mathbb{I}_{B_{R}^{n}}\left(Y_{t / 2}\right)\right] .
\end{aligned}
$$

On the other hand, by 23, 24 and our choice of $R$, we have

$$
\begin{aligned}
\mathbb{E}_{0,0}^{X, Y} & {\left[\exp \left(\int_{0}^{t / 2} I_{p}\left(X_{s}, Y_{s}\right) d s\right) \mathbb{I}_{\left(B_{R}^{p} \times B_{R}^{n}\right)^{c}}\left(X_{t / 2}, Y_{t / 2}\right)\right] } \\
& \leq \exp \left(\frac{t n p}{2}\right) \mathbb{P}_{0}\left(X_{t / 2} \notin B_{R}^{p}\right) \mathbb{P}_{0}\left(Y_{t / 2} \notin B_{R}^{n}\right) \\
& \leq n p \exp \left[\frac{t n p}{2}-(C(d, \rho)+C(d, \kappa)) t \log (t)\right],
\end{aligned}
$$

and therefore, with a similary reasoning as in the proof of Lemma 2.1 we get

$$
\liminf _{t \rightarrow \infty} \frac{1}{t} \log \mathbb{E}_{0}^{\otimes n}\left[u(0, t)^{p}\right] \geq \liminf _{t \rightarrow \infty} \frac{2}{t} \log \mathbb{E}_{0,0}^{X, Y}\left[\exp \left(\int_{0}^{t / 2} I_{p}\left(X_{s}, Y_{S}\right) d s\right)\right]
$$

Now, the occupation measure $\frac{1}{t} \int_{0}^{t} \delta_{\left(X_{s}, Y_{S}\right)} d s$ satisfies a weak large deviations principle (LDP) in the space $\mathscr{M}_{1}\left(\mathbb{Z}^{d p} \times \mathbb{Z}^{d n}\right)$ of probability measures on $\mathbb{Z}^{d p} \times \mathbb{Z}^{d n}$, endowed with the weak topology. The speed of this LDP is $t$ and the rate function is given for all $v \in \mathscr{M}_{1}\left(\mathbb{Z}^{d p} \times \mathbb{Z}^{d n}\right)$ by

$$
J(v)=\kappa\left\|\nabla_{x} \sqrt{v}\right\|_{2}^{2}+\rho\left\|\nabla_{y} \sqrt{v}\right\|_{2}^{2}
$$

(see e.g. den Hollander [12], Section IV.4). Since $I$ is bounded, the lower bound in Varadhan's integral lemma (see e.g. den Hollander [12], Section III.3) yields 


$$
\liminf _{t \rightarrow \infty} \frac{1}{t} \log \mathbb{E}_{0}^{\otimes n}\left[u(0, t)^{p}\right] \geq \sup _{v \in \mathscr{M}_{1}\left(\mathbb{Z}^{d p} \times \mathbb{Z}^{d n}\right)}\left\{\sum_{(x, y)} I_{p}(x, y) v(x, y)-J(v)\right\} .
$$

Setting $f(x, y)=\sqrt{v}(x, y)$ gives then the lower bound in (20).

Step 3: Properties of $\lambda_{p}^{(n)}$. Since $0 \leq I_{p}(x, y) \leq n p$, we clearly have $0 \leq \lambda_{p}^{(n)} \leq n$. Using representation (20), we can conclude that the function $(\kappa, \rho) \mapsto \lambda_{p}^{(n)}(\kappa, \rho)$ is convex and non-increasing in $\kappa$ and $\rho$. Moreover, $\lambda_{p}^{(n)}(\kappa, \rho)$ is lower semicontinuous since it is supremum of functions that are linear in $\kappa$ and $\rho$. Finally, since every finite convex function is also upper semi-continuous, $\lambda_{p}^{(n)}$ is upper semicontinuous. Hence, $\lambda_{p}^{(n)}(\kappa, \rho)$ is continuous.

\section{Proof of Theorems $1.2-1.3$}

By symmetry, note that for all $n, p \in \mathbb{N}$ and $\kappa, \rho \in[0, \infty)$,

$$
\lambda_{p}^{(n)}(\kappa, \rho)=\frac{n}{p} \lambda_{n}^{(p)}(\rho, \kappa)
$$

\subsection{Proof of Theorem 1.2}

Proof of (i): By continuity, $\lim _{\kappa \rightarrow 0} \lambda_{p}^{(n)}(\kappa, \rho)=\lambda_{p}^{(n)}(0, \rho)$. Now for $\kappa=0$, the $X$ particles do not move so that $\mathbb{E}_{0}^{\otimes n}\left[u(0, t)^{p}\right]=\mathbb{E}_{0}\left(\exp \left(p L_{t}^{Y}(0)\right)\right)^{n}$ (see (19)), where $L_{t}^{Y}(0)$ is the local time at 0 of a simple random walk in $\mathbb{Z}^{d}$ with rate $2 d \rho$. Using the LDP for $L_{t}^{Y}$, we obtain

$$
\lambda_{p}^{(n)}(0, \rho)=\frac{n}{p} \sup _{\substack{f \in L^{(2}\left(\mathbb{Z}^{d}\right) \\\|f\|_{2}=1}}\left\langle f,\left(\rho \Delta+p \delta_{0}\right) f\right\rangle=n \mu(\rho / p) .
$$

Proof of (ii): For all $n, p \in \mathbb{N}$ and $\kappa, \rho \in[0, \infty)$, we have

$$
\lambda_{p}^{(n)}(\kappa, \rho) \geq \lambda_{1}^{(n)}(\kappa, \rho)=n \lambda_{n}^{(1)}(\rho, \kappa) \geq n \lambda_{1}^{(1)}(\rho, \kappa)=n \mu(\kappa+\rho),
$$

where the last equality is proved in [3] and comes from the fact that $X_{t}^{1}-Y_{t}^{1}$ is a simple random walk in $\mathbb{Z}^{d}$ with jump rate $2 d(\kappa+\rho)$. Since $G_{d}(0)=\infty$ for $d=1,2$, it follows from (9) that $\lambda_{p}^{(n)}(\kappa, \rho)>0$ for $d=1,2$.

Let us prove that $\lim _{\kappa \rightarrow \infty} \lambda_{p}^{(n)}(\kappa, \rho)=0$. By monotonicity in $\rho$,

$$
\lambda_{p}^{(n)}(\kappa, \rho) \leq \lambda_{p}^{(n)}(\kappa, 0)=n \mu(\kappa / n) .
$$


Hence the only thing to prove is that $\lim _{\kappa \rightarrow \infty} \mu(\kappa)=0$. To this end, one can use the discrete Gagliardo-Nirenberg inequality: there exists a constant $C$ such that for all $f: \mathbb{Z}^{d} \mapsto \mathbb{R}$

$$
\begin{aligned}
& \text { for } d=1,\|f\|_{\infty}^{2} \leq C\|f\|_{2}\|\nabla f\|_{2} ; \\
& \text { for } d=2,\|f\|_{4}^{2} \leq C\|f\|_{2}\|\nabla f\|_{2} .
\end{aligned}
$$

The proof of these inequalities follows the same lines as the proof of the usual Gagliardo-Nirenberg inequality (see e.g. Brezis [1]). For completeness a short proof is given in the appendix. From (27) and (28), we get for all $f \in l_{2}\left(\mathbb{Z}^{d}\right)$ with $\|f\|_{2}=1$,

$$
\begin{aligned}
-\kappa\|\nabla f\|_{2}^{2}+f(0)^{2} & \leq\left\{\begin{array}{l}
-\kappa\|\nabla f\|_{2}^{2}+\|f\|_{\infty}^{2} \text { for } d=1 \\
-\kappa\|\nabla f\|_{2}^{2}+\|f\|_{4}^{2} \text { for } d=2
\end{array}\right. \\
& \leq-\kappa\|\nabla f\|_{2}^{2}+C\|\nabla f\|_{2} .
\end{aligned}
$$

Taking the supremum over $f$ yields

$$
\mu(\kappa) \leq \sup _{x \geq 0}\left(-\kappa x^{2}+C x\right)=\frac{C^{2}}{4 \kappa} .
$$

The strict monotonicity is now an easy consequence of the fact that $\kappa \mapsto \lambda_{p}^{(n)}(\kappa, \rho)$ is convex, positive, non increasing, and tends to 0 as $\kappa \rightarrow \infty$.

Proof of (iii): By (25) and (26), we get

$$
\lambda_{p}^{(n)}(\kappa, \rho) \leq n \min (\mu(\kappa / n), \mu(\rho / p)) .
$$

Then the claim follows by 9 .

\subsection{Proof of Theorem 1.3}

Proof of (i): Fix $\varepsilon>0$. Let $f$ approaching the supremum in the variational representation (20) of $\lambda_{p}^{(n)}(\kappa, 0)$, so that

$$
\begin{aligned}
p \lambda_{p}^{(n)}(\kappa, 0)-\varepsilon & \leq-\kappa\left\|\nabla_{x} f\right\|_{2}^{2}+\sum_{x \in \mathbb{Z}^{d p} p} \sum_{y \in \mathbb{Z}^{d n}} I_{p}(x, y) f^{2}(x, y) \\
& \leq p \lambda_{p}^{(n)}(\kappa, \rho)+\rho \sup _{\substack{f \in l^{2}\left(\mathbb{Z}^{d p} \times \mathbb{Z}^{d n)} \\
\|f\|_{2}=1\right.}}\left\|\nabla_{y} f\right\|_{2}^{2} .
\end{aligned}
$$

For $x \in \mathbb{Z}^{d p}$, set $f_{x}: y \in \mathbb{Z}^{d n} \mapsto f(x, y)$. Since the bottom of the spectrum of $\Delta$ in $l^{2}\left(\mathbb{Z}^{d n}\right)$ is $-4 d n$, 


$$
\sum_{y \in \mathbb{Z}^{d n}}\left\|\nabla_{y} f_{x}(y)\right\|_{2}^{2} \leq 4 d n \sum_{y \in \mathbb{Z}^{d n}} f_{x}^{2}(y),
$$

for all $x \in \mathbb{Z}^{d p}$. Hence,

$$
\sum_{x \in \mathbb{Z}^{d p}} \sum_{y \in \mathbb{Z}^{d n}}\left\|\nabla_{y} f_{x}(y)\right\|_{2}^{2} \leq 4 d n \sum_{x \in \mathbb{Z}^{d p}} \sum_{y \in \mathbb{Z}^{d n}} f_{x}^{2}(y)=4 d n .
$$

Therefore, for all $\varepsilon>0$,

$$
p \lambda_{p}^{(n)}(\kappa, 0)-\varepsilon \leq p \lambda_{p}^{(n)}(\kappa, \rho)+4 d n \rho .
$$

Letting $\varepsilon \rightarrow 0$ yields,

$$
\lambda_{p}^{(n)}(\kappa, 0)-\frac{4 d n \rho}{p} \leq \lambda_{p}^{(n)}(\kappa, \rho) \leq \lambda_{p}^{(n)}(\kappa, 0),
$$

which, after letting $p \rightarrow \infty$, gives the claim.

Proof of (ii): By (25), $\lim _{n \rightarrow \infty} \lambda_{p}^{(n)}(\kappa, \rho)=\lim _{n \rightarrow \infty} \frac{n}{p} \lambda_{n}^{(p)}(\rho, \kappa)$ and by (i),

$$
\lim _{n \rightarrow \infty} \lambda_{n}^{(p)}(\rho, \kappa) \geq \lambda_{n}^{(p)}(\rho, 0)=p \mu(\rho / p)>0, \text { for } p>\rho / G_{d}(0) .
$$

Hence, for $p>\rho / G_{d}(0), \lim _{n \rightarrow \infty} \lambda_{p}^{(n)}(\kappa, \rho)=+\infty$.

Proof of (iii): This is a direct consequence of Theorem 1.2(iii).

\section{Proof of Theorem 1.4}

Proof of (i): We first prove that

$$
\kappa_{p}^{(n)}(\rho)=\sup _{\substack{f \in l_{2}\left(\mathbb{Z}^{d p} \times \mathbb{Z}^{d n}\right) \\\|f\|_{2}=1}} \frac{\sum_{x, y} I_{p}(x, y) f^{2}(x, y)-\rho\left\|\nabla_{y} f\right\|_{2}^{2}}{\left\|\nabla_{x} f\right\|_{2}^{2}},
$$

with $I$ defined as in (17). Indeed, let us denote by $S$ the supremum in the right-hand side of (31).

If $\kappa \geq \kappa_{p}^{(n)}(\rho)$, then $\lambda_{p}^{(n)}(\kappa, \rho)=0$. Therefore, using (20), for all $f \in l_{2}\left(\mathbb{Z}^{d p} \times\right.$ $\left.\mathbb{Z}^{d n}\right)$ such that $\|f\|_{2}=1$,

$$
\sum_{x \in \mathbb{Z}^{d p}} \sum_{y \in \mathbb{Z}^{d n}} I_{p}(x, y) f^{2}(x, y)-\rho\left\|\nabla_{y} f\right\|_{2}^{2} \leq \kappa\left\|\nabla_{x} f\right\|_{2}^{2},
$$


so that $\kappa \geq S$. Hence $\kappa_{p}^{(n)}(\rho) \geq S$. On the opposite direction, we can assume that $S<\infty$. Then, by definition of $S$, for all $f \in l_{2}\left(\mathbb{Z}^{d p} \times \mathbb{Z}^{d n}\right)$ such that $\|f\|_{2}=1$,

$$
\sum_{x \in \mathbb{Z}^{d p}} \sum_{y \in \mathbb{Z}^{d n}} I_{p}(x, y) f^{2}(x, y)-\rho\left\|\nabla_{y} f\right\|_{2}^{2} \leq S\left\|\nabla_{x} f\right\|_{2}^{2} .
$$

Thus, for all $f \in l_{2}\left(\mathbb{Z}^{d p} \times \mathbb{Z}^{d n}\right)$ such that $\|f\|_{2}=1$, and all $\kappa \geq S$,

$$
\sum_{x \in \mathbb{Z}^{d p}} \sum_{y \in \mathbb{Z}^{d n}} I_{p}(x, y) f^{2}(x, y)-\rho\left\|\nabla_{y} f\right\|_{2}^{2}-\kappa\left\|\nabla_{x} f\right\|_{2}^{2} \leq(S-\kappa)\left\|\nabla_{x} f\right\|_{2}^{2} \leq 0 .
$$

Hence, for all $\kappa \geq S, \lambda_{p}^{(n)}(\kappa, \rho)=0$, i.e., $\kappa \geq \kappa_{p}^{(n)}(\rho)$. Hence, $S \geq \kappa_{p}^{(n)}(\rho)$. This proves 31.

Since $\rho \mapsto \kappa_{p}^{(n)}(\rho)$ is a supremum of linear functions, it is lower semi-continuous and convex. It is also obvious that $\rho \mapsto \kappa_{p}^{(n)}(\rho)$ is non increasing. The continuity follows then from the finiteness of $\kappa_{p}^{(n)}(\rho)$.

The lower bound in (13) is a direct consequence of (30). Indeed, since $\lambda_{p}^{(n)}(\kappa, 0)$ $=n \mu(\kappa / n)$, it follows from (30) that if $\mu(\kappa / n)>4 d \rho / p$, then $\kappa<\kappa_{p}^{(n)}(\rho)$. This yields the bound:

$$
\kappa_{p}^{(n)}(\rho) \geq n \mu^{-1}(4 d \rho / p) .
$$

Using the symmetry relation $(25)$, we also get from (30) that

$$
\lambda_{p}^{(n)}(\kappa, \rho) \geq n \mu(\rho / p)-4 d \kappa .
$$

This leads to $\kappa_{p}^{(n)}(\rho) \geq \frac{n}{4 d} \mu(\rho / p)$. Hence, if $\rho / p<G_{d}(0), \kappa_{p}^{(n)}(\rho)>0$. We have already seen that $\kappa_{p}^{(n)}(\rho)=0$ if $\rho / p \geq G_{d}(0)$. Since $\lambda_{p}^{(n)}(\kappa, 0)=n \mu(\kappa / n)$, it follows that $\kappa_{p}^{(n)}(0)=n G_{d}(0)$. Using convexity, we have, for all $\rho \in\left[0, p G_{d}(0)\right]$,

$$
\kappa_{p}^{(n)}(\rho) \leq \frac{\kappa_{p}^{(n)}\left(p G_{d}(0)\right)-\kappa_{p}^{(n)}(0)}{p G_{d}(0)} \rho+\kappa_{p}^{(n)}(0)=n\left(G_{d}(0)-\rho / p\right)
$$

Since $\kappa_{p}^{(n)}(\rho)=0$ if $\rho / p \geq G_{d}(0)$, then the upper bound in 13 is proved.

Proof of (ii): To prove (14), let $f_{0}$ be the function

$$
f_{0}(x, y)=\prod_{i=1}^{p} \frac{G_{d}\left(x_{i}\right)}{\left\|G_{d}\right\|_{2}} \prod_{j=1}^{n} \delta_{0}\left(y_{j}\right) .
$$

Note that for $d \geq 5,\left\|G_{d}\right\|_{2}<\infty$, so that $f_{0}$ is well-defined, and has $l_{2}$-norm equal to 1. From (31), we get

$$
\kappa_{p}^{(n)}(\rho) \geq \frac{\sum_{x, y} I_{p}(x, y) f_{0}^{2}(x, y)-\rho\left\|\nabla_{y} f_{0}\right\|_{2}^{2}}{\left\|\nabla_{x} f_{0}\right\|_{2}^{2}} .
$$


An easy computation then gives

$$
\begin{gathered}
\sum_{x, y} I_{p}(x, y) f_{0}^{2}(x, y)=n p \frac{G_{d}^{2}(0)}{\left\|G_{d}\right\|_{2}^{2}}, \\
\left\|\nabla_{y} f_{0}\right\|_{2}^{2}=n\left\|\nabla_{y_{1}} \delta_{0}\right\|_{2}^{2}=2 d n,
\end{gathered}
$$

and

$$
\left\|\nabla_{x} f_{0}\right\|_{2}^{2}=p \frac{\left\|\nabla_{x_{1}} G_{d}\right\|_{2}^{2}}{\left\|G_{d}\right\|_{2}^{2}}=p \frac{G_{d}(0)}{\left\|G_{d}\right\|_{2}^{2}}
$$

since $\left\|\nabla_{x_{1}} G_{d}\right\|_{2}^{2}=\left\langle G_{d},-\Delta G_{d}\right\rangle=\left\langle G_{d}, \delta_{0}\right\rangle=G_{d}(0)$. This gives (14).

Proof of (iii): The inequality (15) is clear if $\rho \in\left[(p-1) G_{d}(0), p G_{d}(0)\right)$, since in this case, $\kappa_{p-1}^{(n)}(\rho)=0<\kappa_{p}^{(n)}(\rho)$. We assume therefore that $\rho \in\left(0,(p-1) G_{d}(0)\right)$. From (13), we have $\kappa_{p-1}^{(n)}(\rho) \leq n G_{d}(0)-\rho n /(p-1)$, whereas, from (14), $\kappa_{p}^{(n)}(\rho) \geq$ $n G_{d}(0)-\rho n /\left(p \alpha_{d}\right)$. Hence $\kappa_{p-1}^{(n)}(\rho)<\kappa_{p}^{(n)}(\rho)$ as soon as $\alpha_{d}>\frac{p-1}{p}$. This gives the claim.

\section{Proof of Corollary 1.1}

Proof of (i): The function $p \mapsto \lambda_{p}^{(n)}(\kappa, \rho)$ increases from $\lambda_{1}^{(n)}(\kappa, \rho)$ to $n \mu(\kappa / n)$. Hence, there exists $p$ such that $\lambda_{p}^{(n)}(\kappa, \rho)<\lambda_{p+1}^{(n)}(\kappa, \rho)$ as soon as $\lambda_{1}^{(n)}(\kappa, \rho)<$ $n \mu(\kappa / n)$. But $n \mu(\kappa / n)=\lambda_{1}^{(n)}(\kappa, 0)$. Hence, if $\lambda_{1}^{(n)}(\kappa, \rho)=n \mu(\kappa / n)$, the convex decreasing function $\rho \mapsto \lambda_{1}^{(n)}(\kappa, \rho)$ is constant. Being equal to 0 for $\rho \geq G_{d}(0)$, we get that $n \mu(\kappa / n)=0$, which can not be the case if $\kappa<n G_{d}(0)$. This ends the proof of the first part.

If $\kappa \geq n G_{d}(0)$, then $\lambda_{p}^{(n)}(\kappa, \rho)=0$, for all $p \geq 1$, and the system is not intermittent. This proves the second part.

Proof of (ii): For all $p \in \mathbb{N} \backslash\{1\}$ by Lemma 1.1 for $d$ large enough we have $\alpha_{d}>$ $\frac{p-1}{p}$. This implies that $\alpha_{d}>\frac{q-1}{q}$ for all $q \in \mathbb{N} \backslash\{1\}$ and $q \leq p$. Hence, by Theorem 1.4(iii), for all $q \in \mathbb{N} \backslash\{1\}$ with $q \leq p$ we have $\kappa_{q-1}^{(n)}(\rho)<\kappa_{q}^{(n)}(\rho)$, for all $\rho \in$ $\left(0, p G_{d}(0)\right)$. Hence, in the domain

$$
\left\{(\kappa, \rho): \rho \in\left(0, q G_{d}(0)\right), \kappa_{q-1}^{(n)}(\rho) \leq \kappa<\kappa_{q}^{(n)}(\rho)\right\}
$$

one has

$$
\lambda_{1}^{(n)}(\kappa, \rho)=\cdots=\lambda_{q-1}^{(n)}(\kappa, \rho)=0<\lambda_{q}^{(n)}(\kappa, \rho),
$$

which proves the desired result. 
Acknowledgements The research in this paper was supported by the ANR-project MEMEMO.

\section{Appendix: Proof of lemma 1.1}

For a function $f: \mathbb{Z}^{d} \mapsto \mathbb{R}$, let $\hat{f}$ denote the Fourier transform of $f$ :

$$
\hat{f}(\theta)=\sum_{x \in \mathbb{Z}^{d}} e^{i\langle\theta, x\rangle} f(x) \quad \forall \theta \in[0,2 \pi]^{d} .
$$

Then, the inverse Fourier transform is given by

$$
f(x)=\frac{1}{(2 \pi)^{d}} \int_{[0,2 \pi]^{d}} e^{-i\langle\theta, x\rangle} \hat{f}(\theta) d \theta,
$$

and the Plancherel's formula reads

$$
\sum_{x \in \mathbb{Z}^{d}} f^{2}(x)=\frac{1}{(2 \pi)^{d}} \int_{[0,2 \pi]^{d}}|\hat{f}(\theta)|^{2} d \theta .
$$

Using the equation $\Delta G_{d}=-\delta_{0}$ we get that

$$
\hat{G}_{d}(\theta)=\frac{1}{2 \sum_{i=1}^{d}\left(1-\cos \left(\theta_{i}\right)\right)} .
$$

Hence,

$$
\begin{aligned}
G_{d}(0) & =\frac{1}{(2 \pi)^{d}} \int_{[0,2 \pi]^{d}} \frac{d \theta}{2 \sum_{i=1}^{d}\left(1-\cos \left(\theta_{i}\right)\right)} \\
& =\frac{1}{\pi^{d}} \int_{[0, \pi]^{d}} \frac{d \theta}{2 \sum_{i=1}^{d}\left(1-\cos \left(\theta_{i}\right)\right)} \\
& =\mathbb{E}\left[\frac{1}{2 \sum_{i=1}^{d}\left(1-\cos \left(\Theta_{i}\right)\right)}\right]
\end{aligned}
$$

where the random variables $\left(\Theta_{i}\right)$ are i.i.d. with uniform distribution on $[0, \pi]$. Moreover, by Plancherel's formula we have

$$
\left\|G_{d}\right\|_{2}^{2}=\frac{1}{(2 \pi)^{d}} \int_{[0,2 \pi]^{d}} \frac{d \theta}{\left(2 \sum_{i=1}^{d}\left(1-\cos \left(\theta_{i}\right)\right)\right)^{2}}=\mathbb{E}\left[\frac{1}{\left(2 \sum_{i=1}^{d}\left(1-\cos \left(\Theta_{i}\right)\right)\right)^{2}}\right] .
$$

Thus,

$$
\alpha_{d}=\frac{G_{d}(0)}{2 d\left\|G_{d}\right\|_{2}^{2}}=\frac{\mathbb{E}\left[\frac{1}{\bar{S}_{d}}\right]}{\mathbb{E}\left[\frac{1}{\bar{S}_{d}^{2}}\right]}
$$


where $\bar{S}_{d}=\frac{1}{d} \sum_{i=1}^{d}\left(1-\cos \left(\Theta_{i}\right)\right)$. Applying Hölder's and Jensen's inequality, we get that

$$
\alpha_{d} \leq \frac{1}{\sqrt{\mathbb{E}\left[\frac{1}{\bar{S}_{d}^{2}}\right]}} \leq \mathbb{E}\left(\bar{S}_{d}\right)=1 .
$$

By the law of large numbers, $\bar{S}_{d}$ converges almost surely to $\mathbb{E}[1-\cos (\Theta)]=1$ as $d$ tends to infinity. We are now going to prove that $\bar{S}_{d}^{-2}$ is uniformly integrable by showing that for all $p>2$,

$$
\sup _{d>2 p} \mathbb{E}\left[\bar{S}_{d}^{-p}\right]<\infty .
$$

Indeed, let $\varepsilon \in(0, \pi)$ be a small positive number to be fixed later. Let

$$
\begin{gathered}
\mathscr{I}=\left\{i \in\{1, \cdots, d\}: 0 \leq \Theta_{i} \leq \varepsilon\right\} . \\
\bar{S}_{d} \geq \frac{1}{d} \sum_{i \notin \mathscr{I}}(1-\cos (\varepsilon))+\frac{c_{\mathcal{\varepsilon}}}{d} \sum_{i \in \mathscr{I}} \Theta_{i}^{2},
\end{gathered}
$$

where $c_{\varepsilon}=\inf _{0 \leq \theta \leq \varepsilon} \frac{1-\cos (\theta)}{\theta^{2}} \rightarrow 1 / 2$ when $\varepsilon \rightarrow 0$. Therefore,

$$
\mathbb{E}\left[\bar{S}_{d}^{-p}\right] \leq d^{p} \sum_{k=0}^{d} \sum_{\substack{I<\{1, \ldots, d\} \\|I|=k}} \mathbb{E}\left[\frac{\mathbb{I}_{\mathscr{I}=I}}{\left((1-\cos (\varepsilon))(d-k)+c_{\varepsilon} \sum_{i \in I} \Theta_{i}^{2}\right)^{p}}\right] .
$$

Since the last expectation only depends on $|I|$, we get

$$
\mathbb{E}\left[\bar{S}_{d}^{-p}\right] \leq d^{p} \sum_{k=0}^{d}\left(\begin{array}{l}
d \\
k
\end{array}\right) a(k, \varepsilon, d),
$$

with

$$
a(k, \varepsilon, d):=\frac{1}{\pi^{d}} \int_{\substack{0 \leq \theta_{1}, \cdots, \theta_{k} \leq \varepsilon \\ \varepsilon \leq \theta_{k+1}, \cdots, \theta_{d} \leq \pi}} \frac{d \theta_{1} \cdots d \theta_{d}}{\left((1-\cos (\varepsilon))(d-k)+c_{\varepsilon}\left(\theta_{1}^{2}+\cdots+\theta_{k}^{2}\right)\right)^{p}} .
$$

Let $\omega_{d}$ denote the volume of the $d$-dimensional unit ball. For $k=d$,

$$
\begin{aligned}
a(d, \varepsilon, d) & =\frac{1}{\pi^{d}} \int_{0 \leq \theta_{1}, \cdots, \theta_{d} \leq \varepsilon} \frac{d \theta_{1} \cdots d \theta_{d}}{c_{\varepsilon}^{p}\|\theta\|^{2 p}} \\
& \leq \frac{1}{c_{\varepsilon}^{p} \pi^{d}} \omega_{d} \int_{0}^{\sqrt{d} \varepsilon} r^{d-2 p-1} d r \\
& =\left(\frac{\varepsilon}{\pi}\right)^{d} \frac{1}{\left(c_{\varepsilon} \varepsilon^{2}\right)^{p}} d^{\frac{d}{2}-p} \frac{\omega_{d}}{d-2 p},
\end{aligned}
$$

for $d>2 p$.

Note that for large $d, \omega_{d} \simeq \frac{(2 e \pi)^{d / 2}}{\sqrt{\pi d} d^{d / 2}}$. Therefore, as $d \rightarrow \infty$ 


$$
d^{p}\left(\begin{array}{l}
d \\
d
\end{array}\right) a(d, \varepsilon, d)=O\left(d^{-3 / 2}\left(\varepsilon^{2} 2 e / \pi\right)^{d / 2}\right)
$$

If $\varepsilon$ is chosen so that $\varepsilon^{2} \leq \pi /(2 e)$, we obtain that $\lim _{d \rightarrow \infty} d^{p}\left(\begin{array}{l}d \\ d\end{array}\right) a(d, \varepsilon, d)=0$.

For $k \leq d-1$,

$$
a(k, \varepsilon, d) \leq \frac{1}{(1-\cos (\varepsilon))^{p}} \frac{1}{(d-k)^{p}}\left(\frac{\varepsilon}{\pi}\right)^{k}\left(1-\frac{\varepsilon}{\pi}\right)^{d-k},
$$

and $d^{p}\left(\begin{array}{l}d \\ k\end{array}\right) a(k, \varepsilon, d) \leq \frac{1}{(1-\cos (\varepsilon))^{p}} \mathbb{E}\left[\mathbb{I}_{N=k}(1-N / d)^{-p}\right]$, where $N$ is a Binomial random variable with parameters $d$ and $\varepsilon / \pi$. Hence, for $\varepsilon<\min (\pi, \sqrt{\pi /(2 e)})$,

$$
\begin{aligned}
& \mathbb{E}\left[\frac{1}{\bar{S}_{d}^{p}}\right] \\
& \leq \frac{1}{(1-\cos (\varepsilon))^{p}} \mathbb{E}\left[\mathbb{I}_{N \leq d-1}(1-N / d)^{-p}\right]+O\left(d^{-3 / 2}\right) \\
& \leq \frac{d^{p}}{(1-\cos (\varepsilon))^{p}} \mathbb{P}\left[d \frac{2 \varepsilon}{\pi} \leq N \leq d-1\right]+\frac{1}{(1-\cos (\varepsilon))^{p}\left(1-\frac{2 \varepsilon}{\pi}\right)^{p}}+O\left(d^{-3 / 2}\right) .
\end{aligned}
$$

Now, by the large deviations principle satisfied by $N / d$, there is an $i(\varepsilon)>0$ such that $\mathbb{P}[N \geq d 2 \varepsilon / \pi] \leq \exp (-d i(\varepsilon))$. This ends the proof of (32).

Using the uniform integrability (32), and the fact that $\bar{S}_{d}$ converges a.s. to 1 , we obtain that $\mathbb{E}\left[\frac{1}{\bar{S}_{d}}\right]$ and $\mathbb{E}\left[\frac{1}{\bar{S}_{d}^{2}}\right]$ both converge to 1 , when $d$ goes to infinity.

\section{Appendix: Proof of proposition 1.1.}

Let $f \in l_{2}\left(\mathbb{Z}^{d(1+n)}\right)$ with $\|f\|_{2}=1$, such that $\mathscr{L}_{1} f=\lambda_{1}^{(n)}(\kappa, \rho) f$. Define

$$
\tilde{f}\left(x_{1}, x_{2}, y\right)=f\left(x_{1}, y\right) f\left(x_{2}, y\right), \quad x_{1}, y_{1} \in \mathbb{Z}^{d}, y \in \mathbb{Z}^{d n} \text {. }
$$

Since

$$
\sum_{x_{1}, x_{2}, y} \tilde{f}^{2}\left(x_{1}, x_{2}, y\right)=\sum_{y}\left(\sum_{x} f^{2}(x, y)\right)^{2} \leq\left(\sup _{y} \sum_{x} f^{2}(x, y)\right)\|f\|_{2}^{2} \leq\|f\|_{2}^{4},
$$

it follows that $\tilde{f}$ is in $l_{2}\left(\mathbb{Z}^{d(2+n)}\right)$. A simple computation yields

$$
\Delta_{x_{1}} \tilde{f}\left(x_{1}, x_{2}, y\right)=f\left(x_{2}, y\right) \Delta_{x} f\left(x_{1}, y\right), \quad \Delta_{x_{2}} \tilde{f}\left(x_{1}, x_{2}, y\right)=f\left(x_{1}, y\right) \Delta_{x} f\left(x_{2}, y\right),
$$

and 


$$
\begin{aligned}
\Delta_{y} \tilde{f}\left(x_{1}, x_{2}, y\right)=f\left(x_{2}, y\right) \Delta_{y} f\left(x_{1}, y\right) & +f\left(x_{1}, y\right) \Delta_{y} f\left(x_{2}, y\right) \\
& +\sum_{z \sim y}\left(f\left(x_{1}, z\right)-f\left(x_{1}, y\right)\right)\left(f\left(x_{2}, z\right)-f\left(x_{2}, y\right)\right) .
\end{aligned}
$$

Since

$$
I_{2} \tilde{f}\left(x_{1}, x_{2}, y\right)=f\left(x_{2}, y\right) I_{1} f\left(x_{1}, y\right)+f\left(x_{1}, y\right) I_{1} f\left(x_{2}, y\right),
$$

(recalling (17), this leads to

$$
\begin{aligned}
& \mathscr{L}_{2} \tilde{f}\left(x_{1}, x_{2}, y\right) \\
& \quad=2 \lambda_{1}^{(n)}(\kappa, \rho) \tilde{f}\left(x_{1}, x_{2}, y\right)+\rho \sum_{z \sim y}\left(f\left(x_{1}, z\right)-f\left(x_{1}, y\right)\right)\left(f\left(x_{2}, z\right)-f\left(x_{2}, y\right)\right)
\end{aligned}
$$

(recalling (16). Therefore

$$
\begin{aligned}
\lambda_{2}^{(n)}(\kappa, \rho)\|\tilde{f}\|_{2}^{2} & \geq \frac{1}{2}\left\langle\tilde{f}, \mathscr{L}_{2} \tilde{f}\right\rangle \\
& =\lambda_{1}^{(n)}(\kappa, \rho)\|\tilde{f}\|_{2}^{2}+\frac{\rho}{2} \sum_{y, z \sim y}\left(\sum_{x} f(x, y)(f(x, z)-f(x, y))\right)^{2} .
\end{aligned}
$$

Note that

$$
\sum_{y, z \sim y}\left(\sum_{x} f(x, y)(f(x, z)-f(x, y))\right)^{2} \geq 0
$$

with equality to 0 if and only if for all $y$ and $z \sim y, \sum_{x} f(x, y)(f(x, z)-f(x, y))=0$. Interchanging the role of $z$ and $y$ yields $\sum_{x}(f(x, z)-f(x, y))^{2}=0$, so that for all $x, y$ and $z \sim y, f(x, z)=f(x, y)$. Hence, for all $x, y, f(x, y)=f(x, 0)$. This is impossible since $\|f\|_{2}=1$. Thus, $\lambda_{2}^{(n)}(\kappa, \rho)>\lambda_{1}^{(n)}(\kappa, \rho)$.

\section{Appendix: Proof of the discrete Gagliardo-Nirenberg inequality.}

Proof for $d=1$. One can assume that $\|f\|_{2}<\infty$, otherwise there is nothing to prove. Hence $\lim _{|x| \rightarrow \infty}|f(x)|=0$, and by the Cauchy-Schwarz inequality, we have for all $x \in \mathbb{Z}$

$$
\begin{aligned}
f^{2}(x) & =\sum_{j=-\infty}^{x} f^{2}(j)-f^{2}(j-1) \\
& \leq \sum_{j=-\infty}^{+\infty}|f(j)-f(j-1)|(|f(j)|+|f(j-1)|) \\
& \leq 2 \sqrt{\sum_{j}|f(j)-f(j-1)|^{2}} \sqrt{\sum_{j} f^{2}(j)} \\
& =2\|f\|_{2}\|\nabla f\|_{2}
\end{aligned}
$$


which proves 27) with $C=2$.

Proof for $d=2$. Here again, one can assume that $\|f\|_{2}<\infty$, and consequently $\lim _{\left|x_{1}\right| \rightarrow \infty}\left|f\left(x_{1}, x_{2}\right)\right|=0$. Then, by the Cauchy-Schwarz inequality, we have for all $x_{1}, x_{2} \in \mathbb{Z}$,

$$
\begin{aligned}
f^{2}\left(x_{1}, x_{2}\right) & =\sum_{j_{1}=-\infty}^{x_{1}} f^{2}\left(j_{1}, x_{2}\right)-f^{2}\left(j_{1}-1, x_{2}\right) \\
& \leq \sum_{j_{1}=-\infty}^{+\infty}\left|f\left(j_{1}, x_{2}\right)-f\left(j_{1}-1, x_{2}\right)\right|\left(\left|f\left(j_{1}, x_{2}\right)\right|+\left|f\left(j_{1}-1, x_{2}\right)\right|\right) \\
& \leq 2 \sqrt{\sum_{j_{1}}\left|f\left(j_{1}, x_{2}\right)-f\left(j_{1}-1, x_{2}\right)\right|^{2}} \sqrt{\sum_{j_{1}} f^{2}\left(j_{1}, x_{2}\right)} \\
& =2\left\|f\left(\cdot, x_{2}\right)\right\|_{2} \sqrt{\sum_{j_{1}}\left|\nabla_{x_{1}} f\left(j_{1}, x_{2}\right)\right|^{2}}:=2 \tilde{f}_{1}\left(x_{2}\right) .
\end{aligned}
$$

Similarly, we have

$$
f^{2}\left(x_{1}, x_{2}\right) \leq 2\left\|f\left(x_{1}, \cdot\right)\right\|_{2} \sqrt{\sum_{j_{2}}\left|\nabla_{x_{2}} f\left(x_{1}, j_{2}\right)\right|^{2}}:=2 \tilde{f}_{2}\left(x_{1}\right) .
$$

Thus

$$
\sum_{x_{1}, x_{2}} f^{4}\left(x_{1}, x_{2}\right) \leq 4\left(\sum_{x_{2}} \tilde{f}_{1}\left(x_{2}\right)\right)\left(\sum_{x_{1}} \tilde{f}_{2}\left(x_{1}\right)\right) .
$$

Since

$$
\begin{aligned}
\sum_{x_{2}} \tilde{f}_{1}\left(x_{2}\right) & =\sum_{x_{2}}\left\|f\left(\cdot, x_{2}\right)\right\|_{2} \sqrt{\sum_{j_{1}}\left|\nabla_{x_{1}} f\left(j_{1}, x_{2}\right)\right|^{2}} \\
& \leq \sqrt{\sum_{x_{2}}\left\|f\left(\cdot, x_{2}\right)\right\|_{2}^{2}} \sqrt{\sum_{x_{2}} \sum_{j_{1}}\left|\nabla_{x_{1}} f\left(j_{1}, x_{2}\right)\right|^{2}} \\
& \leq\|f\|_{2}\|\nabla f\|_{2},
\end{aligned}
$$

and the same being true for $\sum_{x_{1}} \tilde{f}_{2}\left(x_{1}\right)$, it follows that

$$
\|f\|_{4}^{4} \leq 4\|f\|_{2}^{2}\|\nabla f\|_{2}^{2}
$$

which proves (28) with $C=2$. 


\section{References}

1. Brezis H.: Analyse fonctionnelle: Théorie et applications. Collection Mathématiques Appliquées pour la Maîtrise, Masson, Paris (1983)

2. Carmona R.A., Molchanov S.A.: Parabolic Anderson Problem and Intermittency. AMS Memoir 518, American Mathematical Society, Providence RI (1994)

3. Gärtner J., Heydenreich M.: Annealed asymptotics for the parabolic Anderson model with a moving catalyst. Stoch. Proc. Appl. 116, 1511-1529 (2006)

4. Gärtner J., den Hollander F.: Intermittency in a catalytic random medium. Ann. Probab. 34, 2219-2287 (2006)

5. Gärtner J., den Hollander F., Maillard G.: Intermittency on catalysts: symmetric exclusion. Electronic J. Probab. 12, 516-573 (2007)

6. Gärtner J., den Hollander F., Maillard G.: Intermittency on catalysts. In: Blath J., Mörters P., Scheutzow M. (eds.) Trends in Stochastic Analysis, London Mathematical Society Lecture Note Series 353, pp. 235-248. Cambridge University Press, Cambridge (2009)

7. Gärtner J., den Hollander F., Maillard G.: Intermittency on catalysts: three-dimensional simple symmetric exclusion. Electronic J. Probab. 72, 2091-2129 (2009)

8. Gärtner J., den Hollander F., Maillard G.: Intermittency on catalysts: voter model. Ann. Probab. 38, 2066-2102 (2010)

9. Gärtner J., König W.: The parabolic Anderson model. In: Deuschel J.-D., Greven A. (eds.) Interacting Stochastic Systems, pp. 153-179, Springer, Berlin (2005)

10. Gärtner J., Molchanov S.A.: Parabolic problems for the Anderson model. Commun. Math. Phys. 132, 613-655 (1990)

11. Greven A., den Hollander F.: Phase transition for the long-time behavior of interacting diffusions. Ann. Probab. 35, 1250-1306 (2007)

12. den Hollander F.: Large Deviations. Fields Institute Monographs 14, American Mathematical Society, Providence, RI, (2000)

13. Kesten H., Sidoravicius V.: Branching random walk with catalysts. Electr. J. Prob. 8, 1-51 (2003)

14. Maillard G., Mountford T., Schöpfer S.: Parabolic Anderson model with voter catalysts: dichotomy in the behavior of Lyapunov exponents. In this volume.

15. Schnitzler A., Wolff T.: Precise asymptotics for the parabolic Anderson model with a moving catalyst or trap. In this volume. 